\title{
Bridging the gap between wild and laboratory animals
}

\author{
Jennifer Brunner, BS, RLATG, Animal Facility Manager, USGS National \\ Wildlife Health Center, Madison, WI
}

\section{Ms. Brunner discusses the challenges and the rewards of working with wild-caught animals.}

\section{How did you become involved in the field of wildlife disease research?}

I've wanted to work with animals since I was old enough to understand that I would have a job someday. Growing up in rural northern Wisconsin, I connected with the outdoors early on and understood the value of wildlife, both intrinsically and in terms of their benefit to humans. I graduated from the University of Wisconsin-Madison with a degree in zoology, leaning toward working in the area of environmental conservation and research. I didn't think I'd ever work with captive animals. My first job after college involved freshwater mussel research and management at the USGS Leetown Science Center (Kearneysville, WV), working in both wet labs and the field. This really helped me to gain an appreciation for the role that captive animals play in our understanding of the complex relationships within an ecosystem. The several years I spent at the Wisconsin National Primate Research Center fostered my interest in the more traditional laboratory animal arena. Now I have the best of both worlds: my work aims to improve understanding of certain wildlife diseases through research on both traditional laboratory animal models and wild-caught species for eventual application in the field. I feel good knowing that the work we do helps environmental conservation and management efforts. A healthier planet means healthier animals and healthier humans.

Tell us more about your experiences working with the wide variety of animals that come through your facility.
We're fortunate to be a relatively small program, which allows us to tackle tasks from a team perspective. A lot of research goes into developing housing practices for new species of wild animals (some that have rarely, if ever, been kept in captivity previously). The attending veterinarian, the principal investigator and I all collaborate to develop husbandry standard operating procedures, which then go to full committee review by the IACUC. We're obviously concerned with animal welfare first and foremost, but there are unique concerns that arise related to personnel safety, accessibility of animals for sampling and containment of the specific disease agent being used.

For example, Mexican free-tailed bats, which are used in white-nose syndrome research, are potential carriers of rabies. This requires us to vaccinate all staff and provide lots of hands-on training in capture and handling techniques (in addition to helping staff to get over some natural squeamishness around bats). This particular bat species is insectivorous and takes live prey on the wing in the wild, but we conditioned them to eat mealworms from a dish in order to keep the food source, and potentially the disease agent, contained. Bats are nocturnal, so the difficulty of scheduling husbandry and sampling procedures around their periods of activity and rest resulted in our using a non-standard light cycle in the rooms housing them. Additionally, the study using the bats required them to alternate between several months-long periods of activity (during which they were housed in 'escape-proof' flight cages) and periods of torpor (during which they were housed in a custom-made, high-humidity/ low-temperature hibernaculum).

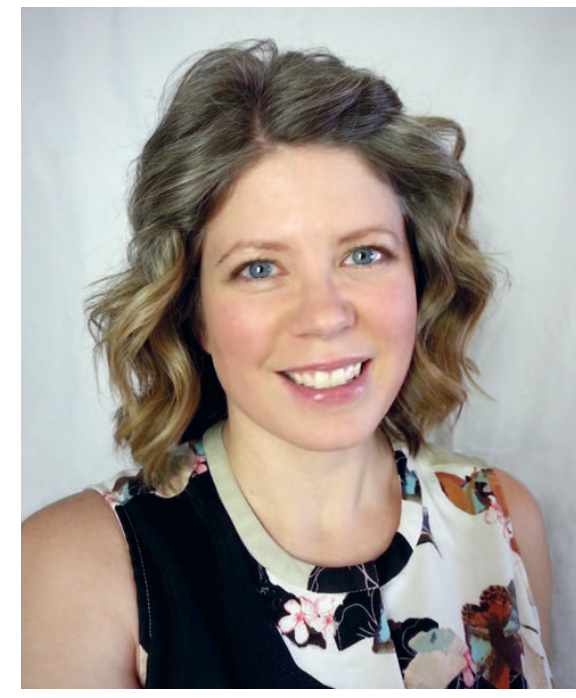

Over the years, we've housed numerous wild-caught species that all raised unique challenges. We try to prepare for every contingency in the beginning, and we learn a lot as we go.

\section{What has been the most challenging aspect of your work?}

Working with wild-caught animals in captivity, we often find ourselves faced with regulatory, veterinary and housing issues that combine aspects of zoo medicine, wildlife management and laboratory animal science. We have a lot of unique species that present husbandry conundrums, and the high animal biosafety level (ABSL-3) of most of our studies adds to the level of difficulty. But I probably struggle with staffing issues more than anything else. Working with laboratory animals is rough work, physically and emotionally, and that results in staff burnout and high turnover. I think most of the people that stay here longterm stick around because they embrace our overall mission of contributing to wildlife and ecosystem health. We do research on wildlife, for wildlife. How great is that? We have a pretty strong team as a result, and my co-workers (especially the animal husbandry techs-you guys rock!) are definitely one of my favorite parts of working here. 